\title{
El movimiento de pobladores chilenos y la población La Victoria: ejemplaridad, movimientos sociales y el derecho a la ciudad
}

Alexis Cortés. Universidade do Estado do Rio de Janeiro, Río de Janeiro, Brasil.

ReSumen | La toma de terrenos de la población La Victoria (Santiago, Chile) ha sido señalada como el marco de inicio del movimiento de pobladores chileno, pues con ella entraron en escena los pobladores y, además, se anticipó un repertorio que se generalizaría en los años setenta. La trayectoria de La Victoria se presenta, por tanto, como una experiencia ejemplar y privilegiada para analizar el desarrollo del propio movimiento de pobladores, así como las principales teorías que buscaron su comprensión: la teoría de la marginalidad, la teoría de los movimientos sociales urbanos y la teoría de los nuevos movimientos sociales. En este artículo se muestra cómo el tratamiento de las ciencias sociales hacia los pobladores ha oscilado entre anuncios de la novedad del movimiento y su réquiem, contrastando estas teorías con algunas categorías de la teoría de las oportunidades políticas y de la geografía crítica, y se señala la necesidad de repensar el movimiento de pobladores a la luz de la experiencia de La Victoria.

PALABras Clave | conflicto social, marginalidad, movimientos sociales.

ABSTRACT | The take-over of land that gave birth to the squatter settlement known as La Victoria (Santiago, Chile) has been considered the starting point of the Chilean pobladores movement, because with it the pobladores came on the scene, anticipating a repertory that became generalized in the 1970s. La Victoria's trajectory appears then as a privileged experience to analyze both the pobladores movement's development and the main theories that seek to understand it: the marginality theory, the urban social movements theory and the new social movements theory. This paper shows how the social sciences treatment of the pobladores oscillated between proclamations of the newness of the movement and its requiem, contrasting theses theories with some categories of the political opportunities theory and the critic geography, and indicating the need to rethink the pobladores movement under the light of La Victoria's experience.

KEY WORDS | social conflict, marginality, social movements.

Recibido el 19 de diciembre 2011, aprobado el 22 de octubre de 2012

E-mail: cortes.alexis@gmail.com

Agradezco a los profesores Gonzalo Cáceres, Breno Bringel y Luiz Antonio Machado por sus estímulos, diálogos y comentarios sobre el tópico aquí abordado, sin los cuales este texto no hubiera sido escrito. Obviamente, cualquier error en el mismo es de mi exclusiva responsabilidad. 


\section{Introducción}

La toma de terrenos de la población La Victoria marcó un antes y un después en la historia urbana de Santiago de Chile, pues si bien los pobres urbanos habitantes de "callampas" existían con anterioridad y el problema habitacional ya estaba presente en la discusión nacional, con esta toma, los "pobladores" se harían visibles como actores sociales, impidiendo que la situación de este grupo social continuara siendo ignorada por el resto de la sociedad.

A pesar de la creencia de los victorianos, la ocupación que dio origen a la población La Victoria no fue la primera toma de terrenos organizada que tuvo lugar en Santiago de Chile (Cáceres, 1993); no obstante, tuvo un impacto público inédito, por lo que es considerada por algunos autores como el hito que dio comienzo a la historia del movimiento de pobladores chileno (Espinoza, 1988; Garcés, 2002). Efectivamente, la toma organizada en 1957 por el Comité de Allegados del Zanjón de la Aguada marca la irrupción de un nuevo sujeto social en el marco de las luchas sociales del país andino y, al mismo tiempo, anticipa un repertorio de lucha popular que alcanzó su punto más alto durante el gobierno de la Unidad Popular (19701973), por la generalización de la toma de terrenos como estrategia de solución del problema habitacional en las principales urbes chilenas (Pastrana \& Threlfall, 1974).

La propuesta de este artículo es analizar la trayectoria del movimiento de pobladores durante el siglo xx, a la luz de la experiencia de este barrio popular. La población La Victoria posee una trayectoria singular; por lo tanto, no es su representatividad lo que nos interesa, ya que en este caso no se aplica, sino más bien su ejemplaridad como uno de los paradigmas de organización del movimiento de pobladores chileno. Este ejercicio de diálogo entre el movimiento general y el caso particular permitirá también revisitar la bibliografía que intentó comprender el movimiento de pobladores desde diferentes ángulos teóricos. De esta manera, a través de la experiencia de La Victoria, podremos repasar brevemente el debate en torno a la marginalidad (Bentué, 1972; Vekemans \& Silva, 1969), así como el principal esfuerzo de comprensión teórica emergido desde la experiencia poblacional, a saber, la teoría de los movimientos sociales urbanos (Centro Interdisciplinario de Desarrollo Urbano [CIDU], 1972; Castells, 1972) y, por consecuencia, las críticas teóricas a la misma ligadas a la perspectiva de los nuevos movimientos sociales (Tironi, 1987b; Touraine, 1987), que cuestionaron la propia condición de movimiento social de los pobladores. Asimismo, la experiencia de La Victoria permitirá levantar algunos temas necesarios para el estudio de la acción colectiva de los pobres urbanos; entre ellos, el movimiento de pobladores y la experiencia de la dictadura, y la crisis y reinvención del movimiento en la posdictadura (Sabatini \& Wormald, 2004).

Si bien existe una literatura consolidada sobre el tema en Chile, también hay tradiciones teóricas con casi nula penetración en el debate nacional sobre pobladores; en particular me refiero a las perspectivas elaboradas por autores como Sidney Tarrow y Charles Tilly (2009) y a la Geografía Crítica (Santos, 2008). Tal vacío ha llevado a que uno de los objetivos de este artículo sea contrastar algunas categorías y conceptos de estas perspectivas con las teorías que han marcado la discusión chilena sobre el movimiento de pobladores, para así repensar este último. 
Para la realización de este artículo se revisó un amplio corpus bibliográfico sobre los pobladores y sobre La Victoria, lo que fue complementado con entrevistas realizadas tanto a pobladores que participaron de la fundación de la misma (veinte entrevistas), como a la generación que vivió el periodo dictatorial (dieciocho entrevistas). Las entrevistas al grupo de fundadores fueron publicadas en el libro Memorias de La Victoria: una aproximación a la historia de la población, por el Grupo Identidad de Memoria Popular (GIMP) (2006), mientras que el segundo bloque fue entrevistado en el marco de la elaboración de mi disertación de maestría en sociología ${ }^{1}$.

\section{La toma de La Victoria}

La primera mitad del siglo xx en Chile estuvo caracterizada, desde el punto de vista urbano, por una alta presión demográfica ejercida por el desplazamiento migratorio desde regiones hacia la capital. La crisis del salitre en el norte, la concentración de tierras en latifundios en el campo y el atractivo dado por un incipiente desarrollo industrial concentró las expectativas de las familias en la ciudad de Santiago. La capital de Chile, en las primeras seis décadas del siglo xx, recibió cerca de un millón de inmigrantes provenientes del resto de las regiones del país. Fue en el periodo 19301950 cuando Santiago experimentó sus tasas más altas de crecimiento urbano, del cual la mitad se debía a migración (De Ramón, 2007).

La ciudad no estaba preparada para recibir ese contingente, por lo que el problema de la vivienda se puso a la orden del día. La primera estrategia de poblamiento popular fue el asentamiento en "conventillos". Sin embargo, la fuerte especulación por el crecimiento desproporcionado de la demanda por habitación rápidamente inviabilizó este mecanismo como única respuesta. Fue así que aparecieron las "poblaciones callampas", ocupaciones de tierra en sectores desfavorecidos (riberas de ríos o zanjones), pero próximos al centro. Según datos proporcionados por Vicente Espinoza (1988), a partir del Censo de Vivienda de 1952, 75.000 personas vivían en callampas para ese entonces, aumentando esta cifra a 150.000 para 1959 (5 y 8 por ciento de la población de Santiago, respectivamente).

De entre estas poblaciones callampas, la más numerosa era la del Zanjón de la Aguada, el llamado "Cordón de la Miseria", con entre cinco y seis mil familias instaladas, unos 35.000 habitantes divididos en diez poblaciones, en una superficie de 5 kilómetros de longitud y 125 metros de ancho, lo que daba una densidad de 640 habitantes por hectárea. Estas familias, que llevaban diez ańos esperando una solución definitiva para su problema de vivienda, sufrieron un par de incendios, en los que "se quemaba hasta la pobreza" (GIMP, 2006, p. 30) y en donde los que

1 En el primer caso, tanto la muestra como la ejecución de las entrevistas fueron realizados por el Grupo Identidad de Memoria Popular, constituido principalmente por jóvenes pobladores (del cual el autor de este artículo formó parte), los que seleccionaron a un grupo de fundadores que participaron en la toma o, al menos, en la instalación del "campamento", buscando mantener un cierto equilibrio en la procedencia organizacional de los entrevistados: comunistas, socialistas, católicos e independientes. Un criterio similar se aplicó en la elaboración de la muestra de la segunda generación, la que vivió la dictadura militar, donde, además, se incorporaron pobladores que militaron en el Movimiento de Izquierda Revolucionaria (MIR). 
nada tenían lo perdían todo, situación que llevó a un grupo de dirigentes locales, en buena parte ligados al Partido Comunista, a elaborar un plan para tomarse los terrenos de la chacra La Feria (Millas, 1996).

Tras algunos ensayos en otros terrenos baldíos, la madrugada del 30 de octubre de 1957, 1.200 familias, bajo la consigna "trabajar sin transar ni descansar, hasta la casa conquistar", con nada más que unos pocos enseres, tres palos y una bandera chilena, se tomaron los terrenos de la chacra La Feria y fundaron el campamento La Victoria (Farías, 1989)².

La toma, como acción directa portadora de una legitimidad basada en la necesidad y en la noción de derecho a la vivienda, en contraposición a la legitimidad procedimental del Estado moderno, fue una acción colectiva organizada que significó, en la práctica, una fractura radical con las lógicas institucionales y con el principio fundamental de las democracias liberales, a saber, la propiedad (Salazar, 2006). Al mismo tiempo, tuvo como consecuencia la inauguración de una nueva esfera de conflictos en la sociedad chilena.

La acción de los pobladores de La Victoria no solo fue un desborde de la institucionalidad y legalidad vigentes; además, hizo visible a un actor social que había permanecido marginado no solo de la esfera pública oficial, sino también del imaginario político de la izquierda chilena, la que centraba su praxis en la clase obrera y en el sindicato. Con esta acción directa, son los pobladores los que "toman su sitio" (Garcés, 2002), lo que implica una nueva dimensión en la configuración de los movimientos sociales en Chile, y también en la articulación del espacio público, en tanto los pobladores emergen como un actor político relevante capaz de poner en tela de juicio al Estado, evidenciando contradicciones al interior de la sociedad chilena que hasta ese momento permanecían invisibles.

Con la toma de terrenos de La Victoria, se anticipó, además, una estrategia de poblamiento popular que se generalizaría en las dos décadas siguientes. La Victoria se transformaba así no solo en una fuente de inspiración simbólica de futuras tomas, sino también en la principal irradiadora de un repertorio de acción colectiva que fue la marca característica del movimiento de pobladores.

Ahora bien, si ya la toma demandó un altísimo nivel de organización, una vez asentados los ocupantes en los terrenos las exigencias organizativas se multiplicaron, toda vez que ahora la tarea era administrar el propio territorio, urbanizarlo sin el apoyo del Estado y defenderlo ante un posible desalojo.

Para el arquitecto Miguel Lawner (2007), quien fue uno de los profesionales comunistas que colaboró con el Comando de Pobladores, uno de los aspectos más relevantes de la toma de La Victoria fue su carácter planificado, ya que, gracias también a una:

Debida asistencia profesional [la ocupación del espacio pudo hacerse de forma ordenada] conforme a trazados urbanísticos normales (...). [En contraste] las tomas anteriores habían obedecido más bien a acciones espontáneas, y si lograban vencer

2 No obstante, según Miguel Lawner -quien, al igual que Guillermina Farías, participó en la toma de terrenos, aunque en calidad de asesor del comando que la dirigió-, el grito coreado era: "La Feria y Lo Valledor... para El Zanjón” (Lawner, 2007, p. 82). 
la represión policial, surgían más tarde obstáculos insuperables para el mejoramiento de la población, a causa de la caótica distribución en el espacio (p. 82).

Aunque recibieron ayuda de algunos profesionales de izquierda, como el propio Lawner, fueron los mismos pobladores los que diseñaron la población, lotearon los terrenos, definieron los espacios públicos y los construyeron. Los pobladores de La Victoria fueron sus propios urbanistas. Para ello, organizaron planes de emulación entre los distintos bloques de la población, premiando y estimulando a aquellos pobladores que más destacaran por su sacrificio en los procesos de colocación de postes eléctricos y de cańerías. También se conformaron comités de vigilancia, encargados de controlar la delincuencia y dar seguridad a la población; se premiaba a aquellos vecinos que tuvieran el jardín mejor cuidado, etcétera. Se creó un periódico interno, La Voz de La Victoria, órgano oficial del Comité Central de Pobladores, que proclamaba su misión como la de ser "el vocero auténtico de las clases trabajadoras que viven y sufren heroicamente, sin desmayar en nuestra población” ( $L a$ Voz de la Victoria, 1[1], 16 de noviembre de 1958, s.p.).

Una de las primeras medidas después de la toma fue crear el Comité Central de Pobladores, que se puso al frente de una serie de bloques, comités y sectores. La finalidad de estos últimos era hacerse cargo del proceso de urbanización sin apoyo público y administrar autónomamente el territorio tomado, bajo criterios amplios de participación de los pobladores.

Lo primero que se hizo fue planificar los terrenos; empezamos con urbanizar la población haciendo todas las calles y dejando terreno para lo que necesitáramos, para la iglesia, los carabineros, las áreas verdes y para la escuela, porque a nosotros no se nos escapó nada; queríamos una población que reuniera todas las cualidades (Eliecer Valenzuela, en Gimp, 2006, p. 91).

Para la instalación eléctrica de la población fue necesario movilizar diversos recursos. Los postes fueron regalados por los pobladores de La Legua, población hermana de la cual provenía parte importante de los fundadores que participaron en la toma. Además, se debieron organizar actividades para comprar los cables. Para la instalación de la electricidad contactaron al ingeniero comunista Enrique Kirberg, quien años más tarde llegaría a ser rector de la Universidad Técnica del Estado.

Para resistir a los intentos de desalojo, además de las medidas de seguridad y de fuerza, fue necesario articular y movilizar a una serie de aliados. Fue así como el cardenal José María Caro intercedió a favor de los pobladores frente al presidente Ibáñez. De la misma manera, incluso desde el día de la toma, fueron convocados los parlamentarios del Frente de Acción Popular, coalición política que integraba a comunistas y socialistas. Los pobladores también contaron con la solidaridad de la Central Única de Trabajadores y de la Federación de Estudiantes de Chile, la que envió voluntarios para ayudar en el proceso de urbanización.

En todo este proceso, fue fundamental el papel que cumplieron cuadros obreros, algunos de ellos con trayectoria en las luchas sindicales del norte minero (Cortés, 2008). En su mayoría militantes del Partido Comunista, estos cuadros traspasaron buena parte de su capital político y de su experiencia organizativa a la toma de La Victoria, coordinando y controlando la dirección del movimiento, desde el Comité 
Central de Pobladores hasta los delegados por cuadras. También protagónico fue el papel de la Iglesia Católica y del Hogar de Cristo, notablemente por la acción del padre Del Corro, director de esta última organización, quien participó de la toma y se instaló desde el primer día en el campamento, incluso llegando a disputarles la conducción a los comunistas, los que finalmente mantuvieron su hegemonía, no sin antes expulsarlo de la población (Barros, 1978).

Si bien el desalojo de la toma no se produjo, se realizó un cerco que impedía la entrada y salida de personas del campamento, además de bloquear el ingreso de materiales de construcción y medicinas. La resistencia de los pobladores generó un alto grado de solidaridad interna, y además permitió la elaboración de una narrativa heroica que encontró sus fuentes en la idea de lucha y organización (Cortés, 2011). Tal como lo señaló un poblador: "Este sitio se le debe a una madre que se llama Organización y a una madre que no se le puede negar la entrada a su casa" (Paiva, 1989, p. 24). La Victoria se consiguió gracias a la lucha:

Siempre quisieron echarnos, sacarnos, venían en la noche con patrullas, con todo para echarnos, pero aquí dijeron no: los niños adelante, las mujeres atrás y los hombres al último, por eso nunca pudieron echarnos, porque la gente era muy unida (Marta Fernández, en Gimp, 2006, p. 53).

\section{Pobladores marginales}

¿Cómo comprender a este nuevo actor social?, ¿Cómo comprender a estos pobres urbanos que irrumpían en la escena pública desafiando las estrategias políticas tradicionales? Sin duda, la comprensión de los pobladores supuso un gran desafío para diversas teorías, las que buscaron dar cuenta de esta nueva realidad y de estos nuevos actores que entraban en escena sin invitación.

Uno de los primeros esfuerzos serios y controvertidos de comprensión fue el realizado por la teoría de la marginalidad (Vekemans \& Silva, 1969), que, desde una perspectiva funcionalista, intentó conceptualizar a los pobladores como marginales. La marginalidad se identificaría con la falta de participación y de pertenencia a la sociedad, siendo característica propia del continente la dicotomía entre una sociedad participante, instalada y hegemónica versus otra sociedad de masas marginales.

En Chile, fue Roger Vekemans quien inició el estudio de este fenómeno en la década de los sesenta desde el Centro para el Desarrollo Económico y Social de América Latina (Desal), siendo clave en la consistencia ideológica que justificaría la actuación democratacristiana hacia ese nuevo sector de la sociedad chilena. En la concepción de este autor: "el mundo marginal (...) es un mundo internamente desintegrado, atomizado, un mundo en el que si se encuentran 'coagulaciones', ellas serán del tipo ghetto, replegadas sobre sí mismas, a la defensiva, y no dispuestas a enfrentarse con la sociedad establecida" (Vekemans \& Silva, 1976, p. 81).

Bajo esta imagen, los pobladores marginales aparecen como portadores de una subcultura que les impide alcanzar niveles de organización y de interés político adecuados para lidiar con el mundo moderno. En el caso de La Victoria, muchas 
de las lecturas iniciales que se hicieron de ella bebían de esta teoría, incluso con investigaciones que intentaron mostrar a La Victoria como un ejemplo de marginalidad (Bentué, 1972).

Aunque las tesis de la marginalidad fueron cuestionadas íntegramente por numerosas investigaciones (Giusti, 1973; Machado da Silva, 1983; Oliven, 1980; Pearlman, 1977; Sabatini, 1981), en la década de los sesenta tenía una gran influencia en la opinión pública y, en buena medida, alimentó los prejuicios sobre los pobladores. No es de extrañar, entonces, que buena parte del discurso y de las prácticas de los pobladores de La Victoria apuntara a deconstruir la imagen de la masa marginal disfuncional, que en este caso se expresaba en la idea de "callampero". Un buen ejemplo de este esfuerzo de limpieza simbólica es la construcción de la "escuela redonda", que fue erigida colectivamente (cada poblador tenía que hacer adobes y donarlos). La construcción de la escuela simbolizaba varias cosas: por un lado, el manifiesto interés por mostrar ante el mundo que el estigma de "callamperos", como sinónimo de incultura e ignorancia, era inaceptable para los pobladores de La Victoria. En la escuela se reflejaba el esfuerzo colectivo que se orientaba a la superación, a la formación y a la educación, valores propios de "gente trabajadora". Asimismo, retrataba el sacrificio y entrega de los pobladores a una causa colectiva que iba más allá de la conquista de una casa. De allí que quienes contribuían con más esfuerzo en la construcción eran celebrados y destacados públicamente.

Por otra parte, el cariz paternalista que acompañaba la intervención propuesta por la Desal en las poblaciones ("hay que enseñarles a los marginales a salir de la pobreza"), contrastaba con el discurso de marcado rechazo a lo asistencial por parte de los líderes de La Victoria. La consigna que mejor refleja ese espíritu es: "Nada por caridad, todo mediante nuestro propio esfuerzo"3 ( La Voz de la Victoria, 1 [5], 7 de noviembre de 1959 , s.p.), lo que muestra una decisión dirigida a hacer ver que los pobladores mismos eran capaces de construir su propia población, y que expresaban en las siguientes palabras: "Hemos aprendido que la lucha organizada, que la lucha revolucionaria deber ser la única manera de obligar a estas autoridades a dar preferencia a las más urgentes necesidades de la población" (La Voz de la Victoria, 2[6], 31 de diciembre de 1959, s.p.).

La idea de los pobladores era mostrarle al país que eran capaces de levantar una población a partir de sus propias capacidades; que con las herramientas de la unidad, la lucha y la organización se podía salir adelante; en sus palabras:

Sabemos perfectamente que triunfaremos para demostrar a Chile entero que no merecemos el nombre de población "callampa", con que en forma denigrante y despectiva muchos ciudadanos que no conocen la miseria y el grave problema habitacional que aqueja al mundo se permiten motejarnos ( $\mathrm{La}$ Voz de la Victoria, 1[1], 1958, s.p.). 


\section{Los pobladores como movimiento social urbano}

Contagiados por el clima de efervescencia social y política reinante en Chile a comienzos de los años setenta, un grupo de investigadores se dedicó al estudio de lo que parecería ser un nuevo actor social potencialmente decisivo en la polarización chilena de la época: los pobladores. Estos intelectuales vieron en los marginados (o en el lumpen-proletariado) "una punta de lanza urbana" -según la expresión de Fanon (2007) - de los procesos de transformación social que experimentaba el continente. El equipo de Estudios Poblacionales del Centro Interdisciplinario de Desarrollo Urbano (CIDU) fue el principal grupo de investigación que articuló la producción de dichos autores. Este núcleo, ligado a la Pontificia Universidad Católica de Chile, intentó comprender la novedad político-social que representaba el movimiento organizado a partir del establecimiento de innumerables campamentos, originados por tomas de terrenos dirigidas por militantes de partidos políticos (Partido Demócrata Cristiano [DC], Partido Comunista [PC], Partido Socialista [Ps] y Movimiento de Izquierda Revolucionaria [MIR]). Para Santa María (1973), el campamento era una organización social y territorial y una forma de crecimiento no planeado del suelo urbano que, en los años setenta, representaba la operación urbana de mayor importancia en la ciudad de Santiago, sea en la forma de asentamiento espontáneo o como toma organizada.

El cIDU reconocía en los campamentos de pobladores una experiencia única de lucha política y de organización urbana. A juicio de sus investigadores, el trazo que dotaba de originalidad a este movimiento era que el proceso de tomas de terrenos estaba fuertemente vinculado a la cuestión del poder. No se trataba solo de la presencia de organizaciones populares de las más variadas al interior de los campamentos, sino también del papel directamente político ejercido por el acto de toma de terrenos y del proceso político experimentado por Chile durante el periodo en que el movimiento de pobladores ganó centralidad (finales de los sesenta y comienzo de los setenta). Fue esta sumatoria de factores lo que, a juicio del cidu (1972), marcó la intervención diferencial de los diversos actores políticos en los campamentos.

Para Castells (1972), lo que caracterizó al movimiento de pobladores fue la articulación, en el marco de la lucha de clases, de la reivindicación urbana y de una estrategia política ligada a la movilización de objetivos basados en el gobierno local, que a su vez permitió el desarrollo de nuevas formas de organización política.

El interés de estos autores estaba dado precisamente por el auge del movimiento de pobladores en la época, centralidad que se explicaba por la coincidencia táctica de tres estrategias políticas opuestas que competían en el específico espacio de los campamentos y poblaciones para dar una salida a la crisis estructural de la habitación popular en Chile: la democratacristiana, la de la izquierda obrera (PC) y la de la izquierda revolucionaria (MIR).

Ahora bien, en la descripción del movimiento de pobladores realizada por el cIDU parece existir una simbiosis entre este nuevo movimiento y la estrategia política desarrollada por el MIR, que, a finales de los sesenta, intentó disputar la hegemonía del movimiento popular a los partidos obreros tradicionales (PC y PS). Fueron los campamentos organizados por el MIR los que concentraron la atención de estos investigadores, 
quienes reconocerían, en el intento de crear microcomunidades revolucionarias, un salto cualitativo en el movimiento poblacional (Castells, 1972).

Una de las más importantes contribuciones de las investigaciones de estos autores fue haber superado la concepción del movimiento de pobladores como un mero auxiliar del movimiento obrero, y otorgarle un estatus político de primera línea como un sector social que sería decisivo a la hora de resolver la polarización política de la sociedad chilena. Al mismo tiempo, la discusión teórica sobre el movimiento de pobladores que proponían reflejaba las disputas políticas de la época en el propio campo intelectual. En un momento de profunda división dentro de la izquierda, estos autores tomaron posición a favor de una estrategia y de un sujeto: la de la izquierda revolucionaria y los pobladores. Con ello se sobredimensionó el alcance y el papel del movimiento de pobladores ligado a la izquierda radical. La acentuada preocupación por la novedad que el movimiento presentaba en el marco de la coyuntura del gobierno de Allende les hizo perder de vista el previo proceso de conformación como sujeto del propio movimiento. La Victoria, en este caso, quedó fuera del interés de estos autores por estar asociada a la estrategia de los comunistas, la que era identificada como una mera praxis electoralista y economicista, no obstante que en La Victoria se anticiparan repertorios que serían leídos como novedad en los años setenta (Vanderschueren, 1971).

Por otro lado, a pesar de resaltar la importancia de las contradicciones en el ámbito urbano, la lucha de los pobladores, independientemente de su valoración como intento original de transformación de la naturaleza de la situación de subordinación en la cual se encontraban (Quevedo \& Sader, 1973), fue conceptualizada en términos de consumo (habitacional). No hay en estos autores, por tanto, una valoración específica de la importancia del espacio para la configuración de las contradicciones sociales. Ciudad y capitalismo, en ciertos momentos, aparecen como términos intercambiables.

\section{La dictadura, los pobladores y La Victoria}

Este proceso ascendente de luchas de los pobladores fue interrumpido por el golpe de Estado que depuso al gobierno de Salvador Allende y que instauró una dictadura militar (1973). El movimiento de pobladores había sido uno de los pilares de apoyo del gobierno derrocado, por lo que la dictadura concentró buena parte de su acción represiva en las poblaciones, tanto de manera selectiva, con la muerte y desaparición de sus principales dirigentes; como de manera colectiva, con invasiones policiales, cercos de poblaciones, detenciones masivas, secuestros, y destrucción y robo de objetos personales y domésticos de los pobladores (Comité de Memoria Histórica, 2005).

Ahora bien, fue precisamente el golpe de Estado lo que puso en tela de juicio parte importante de las tesis del CIDU expuestas en el apartado anterior, abriendo el campo para una lectura más escéptica del potencial del mundo poblacional: "Mirando el presente del sector, resulta difícil creer que movilizaciones de tal magnitud hayan tenido lugar. Las expectativas de una reactivación parecieran desvanecerse frente a la dura realidad del momento presente. (...) Los pobladores parecen optar por soluciones individuales" (Espinoza, 1982, p. 42). 
Aunque algunos estudiosos del fenómeno poblacional, como el anteriormente citado Vicente Espinoza, buscaron llevar a un plano general las prácticas dispersas del movimiento, en parte importante del campo este escepticismo llevó a cuestionar la existencia misma de un movimiento de pobladores:

... es falso -como se pensó en los sesenta- que haya movimientos urbanos de pobladores híper-radicalizados. Este ejército de reserva de la revolución, en efecto, no se movilizó ni el año 64 en Brasil, ni el 66 o 76 en Argentina, ni el año 73 en Uruguay o Chile (Touraine, 1987, p. 219).

Sin embargo, a comienzo de la década de los ochenta, con el inicio de las protestas nacionales en contra del régimen una vez que la oposición salió del desconcierto y consiguió articularse mejor, fueron precisamente las poblaciones los principales focos de resistencia y de descontento frente a la dictadura de Augusto Pinochet. Para Tomás Moulian (2002), las movilizaciones nacionales de protesta (al menos en su primera fase) pueden ser caracterizadas como formas de ebullición social en las que se habría producido un fenómeno catártico de depuración del sentimiento de represión a través de las diferentes prácticas que la oposición utilizó para manifestar su descontento: caceroleos, bocinazos, barricadas, velorios, etcétera. Máxima expresión de esta experiencia catártica fue, para el autor citado, la protesta en la población, en la que confluyeron dos variables: tiempo y espacio, noche y barrio. Con la caída del crepúsculo, en las poblaciones se desarrollarían las manifestaciones más radicales e intensas en contra del régimen. Desde una perspectiva diferente, Gabriel Salazar (2006) también destaca el papel de los pobladores. Esta "revuelta de los pobladores”, según el historiador, abrió una brecha psicológica y política decisiva en el flanco popular de la dictadura. Para otros autores (Pinto, Candina \& Lira, 1999), estas jornadas de protesta tuvieron como consecuencia una territorialización del poder y de la política popular, uno de cuyos efectos más destacados fue poner en el primer plano a los pobladores.

La reacción de la dictadura a estas manifestaciones fue ampliar el alcance de la violencia, lo que significó, según la investigación Tortura en poblaciones del Gran Santiago (1973-1990), del Colectivo de Memoria Histórica José Domingo Cańas (2005), que el espacio territorial identificado con "la población” se transformara en un espacio de tortura y prisión política en sí, por causa de los dispositivos de represión desarrollados por la dictadura y destinados específicamente a controlar y neutralizar a este actor popular.

Para algunos investigadores (Cáceres, 1993), tras un periodo de reflujo y recomposición, los años ochenta marcarían un renacer del movimiento de pobladores. Y aunque nuevamente emergieron lecturas que resaltaban la novedad del movimiento, autores como Cathy Schneider (1990) pusieron el énfasis en la continuidad:

El movimiento de protesta chileno de 1983-1986, si no fue el precursor de un nuevo actor social, o una respuesta inmediata a la crisis económica de 1982, era más que el producto de militantes políticos aislados que operaban fuera de las poblaciones marginales. Más bien, la capacidad de estos barrios urbanos para movilizar una 
resistencia política masiva tras años de severa represión militar, radicaba en la herencia política de décadas de trabajo en la cultura popular y en la formación de una generación de militantes de base hábiles (pp. 224-225).

Esta misma autora observa que las protestas no se distribuyeron homogéneamente en los barrios pobres ni se concentraron en los sectores más afectados por la crisis económica. Por el contrario, cobraron más fuerza en los mismos "barrios rojos", donde la izquierda había consolidado una presencia de larga data. En estos barrios, la cohesión social y la existencia de una diseminada red de militantes permitieron que se superaran las trabas de la clandestinidad y que se movilizara una resistencia a gran escala, una vez que se desató la crisis económica de 1982. Schneider señala al respecto:

Tampoco en estas poblaciones tuvieron los militantes políticos iguales resultados. Aquellas poblaciones que habían sido organizadas "desde arriba", es decir, por militantes externos a la población, encontraron más difícil mantener la resistencia después del 73, en comparación con aquellas manejadas "desde abajo", donde los partidos políticos habían establecido una base orgánica. Esta diferencia se puede ver claramente al comparar las poblaciones que surgieron a raíz de tomas de terreno ilegales dirigidas por el MIR, y aquellas conducidas por el Partido Comunista (p. 226).

Contrariamente a lo que postulaban algunos investigadores del movimiento de pobladores de los años ochenta, que veían la aparición de un "nuevo actor social" que se diferenciaba de la experiencia de los setenta por su constitución autónoma por sobre la influencia partidaria (Oxhorn, 2004), para Schneider la sobrevivencia del Partido Comunista en algunas poblaciones fue consecuencia de un enfoque conducente a la aparición de "intelectuales orgánicos" desde las bases, que permitió la renovación de los dirigentes encarcelados o asesinados. La base del éxito de este partido habría sido la generación de una cultura popular en los años previos al golpe. Para la autora, el Partido Comunista, en su mejor momento, funcionó como una fuente de cultura y de dirección política, que encontró en la represión dictatorial un refuerzo a su ideología y a sus esfuerzos de generación de historias de lucha colectiva.

Asimismo, no se puede dejar de mencionar el papel de la Iglesia Católica en el desarrollo del movimiento de pobladores (Salinas, 1996). La proscripción de la actividad política oficial la llevó a asumir roles y funciones políticas de primera línea (Cancino, 1997), primero a través de la defensa de los Derechos Humanos, pero también sirviendo como una especie de "escudo protector" del mundo poblacional y como principal fuente de apoyo logístico y humano para la reconstrucción del tejido social popular de las poblaciones (Oxhorn, 2004). En otras palabras, la recomposición del movimiento de pobladores durante la dictadura no hubiera sido posible sin el papel de curas y monjas obreros, así como de activistas y educadores populares ligados a la Iglesia que hicieron de la opción preferencial por los pobres la principal guía de su evangelio. Particularmente en La Victoria, esta noción de "evangelio hecho acción" encontró en sacerdotes como Pierre Dubois y André Jarlan dos de sus más insignes representantes (Santapau, 2005).

Todos estos elementos estuvieron presentes en la experiencia de La Victoria durante la dictadura (Grupo de Trabajo de la Victoria, 2007). En primer lugar, por la identificación de la población con el gobierno de Salvador Allende, La Victoria 
fue objeto de fuerte represión tras el golpe militar, incluida la amenaza de bombardeo por parte de la Fuerza Aérea. Una de las entrevistadas, Gloria Rodríguez, 51 años, relata:

Como la toma de terrenos fue mayoritariamente dirigida por gente de izquierda, fue guiada, conducida por el Partido Comunista y por el Partido Socialista, inmediatamente la dictadura tuvo un sesgo con nuestra población, nos acusaron a todos de comunistas. Por lo tanto, fuimos la población que más allanaron, que más reprimieron y nosotros a su vez nos declaramos abiertamente contra la dictadura (en Cortés, 2009, p. 65).

Como ocurrió con el resto del país, durante los primeros años tras el golpe se produjo un reflujo organizacional, debido a la persecución y represión de la que eran víctimas las organizaciones sociales. Estas también debieron quedar en suspensión, puesto que muchos dirigentes y militantes tuvieron que ocultarse ("guardarse") y pasar a la clandestinidad para evitar engrosar las listas de ejecutados o desaparecidos. Sin embargo, como señaló un poblador en una entrevista:

Si tú analizas el tema de la represión, a la población la afectó toda. Aparte de que hubo casos selectivos, la población fue afectada, fue torturada, fue perseguida, hubo una acción direccionada a neutralizar todo tipo de organización en la población, que obviamente fracasaron, pero que nos marcó (Félix Morales, 52 años, en Cortés, 2009, p. 72).

Paulatinamente en La Victoria se produjo una sustitución generacional de los dirigentes, por lo que contingentes jóvenes fueron reemplazando a los dirigentes ahora perseguidos. La primera tarea que los nuevos líderes enfrentaron, según ellos mismos relatan, fue "vencer el miedo", intentando así recomponer el tejido organizacional. En este sentido, la experiencia de la toma fue invocada para resignificar la situación represiva y alimentar la resistencia de los pobladores, trazándose una línea de continuidad entre ambas experiencias. Al mismo tiempo, se produjo una renovación, por innovación, del repertorio de acciones colectivas en la propia población. Así, entre 1980 y 1986 surgieron y se desenvolvieron en La Victoria experiencias como las "ollas comunes", o los "comedores populares" organizados por la Iglesia Católica. En 1981 se creó el Comprando Juntos; posteriormente, al alero del padre Pierre Dubois, se inició la Leche por Cuadras. También en 1981, el Servicio Evangélico para el Desarrollo (Sepade) organizó el primer curso de primeros auxilios, que posteriormente originó los Grupos de Salud Poblacional, orientados a entregar tratamiento de emergencia a los pobladores heridos en las protestas nacionales (Amorós, 2006). El muralismo desarrollado al interior de La Victoria también se volvió un referente como instrumento de protesta y expresión popular, que fue replicado en otras poblaciones. Aunque es probable que todas estas acciones colectivas no hayan sido "inventadas" en La Victoria, su constelación con dosis de masividad le otorgaron a la población un papel central en la difusión de este repertorio a otros espacios populares.

Paralelo a lo anterior, la resistencia de los victorianos implicó que la población fuese reconocida como uno de los bastiones en la lucha contra la dictadura. 
Reaparece, por tanto, la ejemplaridad de su trayectoria. La Victoria nuevamente se transformaba en sinónimo de rebeldía y organización. Un elemento que contribuyó a la construcción de este imaginario fue la gran cantidad de pérdidas humanas que tuvieron los victorianos durante ese periodo. Cada muerte de un poblador era interpretada no como un crimen individual o particular, sino como un crimen contra la colectividad, contra toda la población. Así, cada funeral de un mártir local se volvía un acto de rebelión contra la dictadura. La población se vaciaba, se quedaba sin habitantes, para marchar en dirección al cementerio, lo que obviamente encontraba una respuesta violenta por parte de la dictadura. El asesinato, por una "bala loca" de la policía, del párroco de La Victoria, el sacerdote francés André Jarlan, fue el caso que más impacto público tuvo de todos ellos, llegando a provocar una condena internacional de la dictadura y de su actuar represivo en las poblaciones chilenas (Verdugo, 1985).

"La Victoria era diferente". Reiteradamente es lo que se escucha decir en los testimonios de sus habitantes. Como la población era atacada como un todo, la respuesta organizada o espontánea también se hacía como un todo. Siempre quien protestaba encontraba una puerta abierta para protegerse de la persecución de la policía, "no como en otros lados". Existía una idea de soberanía popular del espacio en La Victoria, que se manifestaba en la disposición innegociable de evitar que las fuerzas represivas entrasen al barrio: "Que les costara entrar a la población, porque era nuestra, nosotros la habíamos tomado" (Blanca Ibarra, 60 años, en Cortés, 2009, p. 76).

\section{Los pobladores, ¿̨movimiento social?}

A pesar de las diversas formas de protesta que los pobladores opusieron a la dictadura, para el ya citado Alain Touraine (1987) la movilización de los pobres urbanos de Santiago no tenía los elementos suficientes para constituir un movimiento social, según los parámetros que el propio autor hizo célebres:

Si me lo permiten, en mi vocabulario diría que no constituye propiamente un movimiento social, sino un movimiento histórico, donde el tema no es manejar los recursos de una sociedad, de un tipo societal, sino manejar el proceso de transformación social cuyo agente central no es una clase dirigente, sino el Estado (p. 221).

Siendo el autor con más influencia en el ámbito de los movimientos sociales en Chile, las palabras de Touraine tuvieron un fuerte eco en el campo local (Dubet, Tironi, Espinoza \& Valenzuela, 1989). Uno de los autores que recogió los argumentos del sociólogo francés fue Eugenio Tironi (1987a), quien puso en tela de juicio la capacidad del movimiento de pobladores para generar una identidad colectiva llamada a perdurar. El movimiento de pobladores sería más un movimiento de militantes que social. Fue precisamente el empleo de las categorías tourainianas lo que llevaron a Tironi (1986) a afirmar:

En el caso que estamos estudiando de los pobladores, nos encontramos primeramente con la desarticulación entre las orientaciones ofensiva (la acción reivindi- 
cativa) y defensiva (la acción comunitaria); y en segundo lugar, con una situación donde uno y otro tipo de acción encuentran obstáculos que las llevan a ambas a un punto crítico. En estas circunstancias parece difícil, por ejemplo, referirse a los pobladores como a un "movimiento social". En efecto, la crisis de la acción reivindicativa y los límites del comunitarismo desembocan en un fenómeno que corresponde bastante aproximadamente a lo que Touraine denomina a veces genéricamente como un "antimovimiento social”, cuya expresión más patente es la violencia (p. 30).

Para otros autores, como Vicente Espinoza (1984), el gran desafío que presentan los pobladores para las ciencias sociales es su propensión a la discontinuidad, visible en estallidos que parecen no tener relación unos con otros. Aunque a lo largo de su obra buscará encontrar en esos retazos dispersos una tipología de la acción colectiva, Espinoza no será ajeno al pesimismo de la época. Así, un década más tarde señala:

La acción de los pobladores, como es distanciada en el tiempo, sin una organización que le otorgue continuidad estratégica, sin formas de acción características, con demandas de la índole más variada, parece una serie de conflictos discontinuos. La percepción de los pobladores como movimiento social no pasaría de ser una ilusión óptica, por la centralidad que le otorga la permeabilidad de las condiciones políticas (Espinoza, 1994, p. 247).

Nuevamente los énfasis en la novedad y protagonismo del mundo popular daban paso, en las ciencias sociales, al diagnóstico de la ausencia del otrora poderoso movimiento o a la elaboración de informes mortuorios. ¿Realmente existieron movimientos sociales urbanos?, ¿ Fueron una ilusión o una realidad transitoria?

La transición democrática chilena les dio más fuerza a las preguntas anteriores, ya que implicó, en pos de la estabilidad institucional, una desmovilización de aquellos sectores que contribuyeron a la derrota electoral de la dictadura en 1988. Con la llegada de la democracia, el movimiento de pobladores nuevamente perdió protagonismo. La mayor parte de los pobres urbanos pasó a ser propietario de una vivienda social otorgada por los programas habitacionales de la Concertación de partidos gobernantes (Ducci, 1997).

Con todo, la comprensión del movimiento de pobladores en Chile osciló entre la novedad y el réquiem del mismo. No obstante, e independientemente de este vaivén, a lo largo del siglo xx el movimiento de pobladores mostró una gran capacidad de metamorfosis y de adaptación. Estos elementos, ¿no nos muestran acaso la necesidad de repensar este movimiento con otras categorías?

Una alternativa que puede ser fructífera para el enriquecimiento conceptual de la comprensión de los movimientos sociales en Chile y, en particular, del movimiento de pobladores, es el empleo, con las mediaciones pertinentes, de algunas de las categorías divulgadas inicialmente por la teoría de los procesos políticos de Charles Tilly y Sidney Tarrow, entre otros (Alonso, 2009), conceptos que, si bien no son para nada nuevos en la discusión latinoamericana, han estado prácticamente ausentes. Lo que se propone aquí no es el reemplazo de una determinada perspectiva teórica por otra, sino más bien la incorporación crítica y selectiva de 
algunos conceptos que pueden ser útiles para superar la tendencia a atribuir al movimiento de pobladores una tendencia a la discontinuidad ${ }^{4}$.

Un movimiento social es una forma de acción compleja, señala Tilly (1995), no un actor ni un grupo, por lo que, a diferencia de los individuos, no experimenta historias naturales (formación, florecimiento, cambio y desaparición). Para este autor, como para Tarrow (2009), la contestación política ocurre cuando oportunidades y restricciones políticas crean incentivos para que actores sociales, sin otros recursos más que la acción colectiva, actúen, mediante repertorios determinados, desafiando a autoridades o a grupos poderosos.

Un concepto que vale la pena destacar es el de estructura de oportunidades, principalmente por las categorías que los autores mencionados derivarán de él. La contestación política es una reacción a cambios en las oportunidades y restricciones a la acción, sean de carácter institucional, material o cultural. La oportunidad política es vista como una dimensión consistente del ambiente político que favorece incentivos para la acción colectiva y que afecta las expectativas de éxito o fracaso de las personas (Tarrow, 2009). En este concepto, destacable como es, la propia idea de oportunidades políticas evidencia el sesgo estructural de la teoría, más aún si se considera que la movilización a menudo es una respuesta defensiva al constreńimiento de oportunidades políticas, y considerando que:

Mobilization does not necessarily depend on expanding opportunities (except in the tautological sense), and such opportunities, when they are important, do not result from some invariant menu of factors, but from situationally specific combinations and sequences of political processes-none of which, in the abstract, has determinate consequences (Goodwin \& Jasper, 1999, p. 39).

De hecho, en Chile el movimiento de pobladores resurgió en medio de la represión dictatorial de Pinochet y se contrajo con la apertura democrática que le siguió, fenómeno que Philip Oxhorn (2004) denominó como la "paradoja del gobierno autoritario".

No obstante, la idea de estructura de oportunidades conduce a una categoría especialmente útil para comprender la trayectoria del movimiento de pobladores. Me refiero al concepto de ciclos de movilización.

Entendo "ciclo de confronto" como uma fase de conflito acentuado que atravessa um sistema social: com uma rápida difusão de ação coletiva de setores mais mobilizados para outros menos mobilizados; com um ritmo rápido de inovação nas formas de confronto; com a criaçáo de quadros interpretativos de açáo coletiva, novos ou transformados; com uma combinação de participação organizada e não-organizada: e com seqüências de fluxos intensificados de informação e de interação entre os

4 Una de las reservas necesaria frente a estos autores, particularmente en sus trabajos más recientes, es la amplitud de su definición de movimiento social, la que los ha llevado a expandir sus intereses de investigaciones a toda forma de contestación política, descuidando a los propios movimientos sociales. Resulta notable constatar que mientras la definición de Alain Touraine restringe de sobremanera las experiencias que se encajan dentro de su definición de movimiento social, en el caso de Tarrow, Tilly y compañía, los movimientos sociales se diluyen en las acciones colectivas de confrontación política. 
desafiantes e as autoridades. Esse confronto tão disseminado produz externalidades que dão aos desafiantes ao menos uma vantagem temporária e permite que superem a fraqueza na sua base de recursos. Ele exige que o Estado monte amplas estratégias de reação que são ou repressivas ou facilitadoras, ou uma combinação de ambas. E ele produz resultados gerais que são mais do que a soma dos resultados de um agregado de eventos desconectado (Tarrow, 2009, p. 182).

La categoría de ciclos de movilización enfatiza la condición procesual de la propia movilización y no la ve como una serie de eventos aislados unos de otros. Bajo esta lógica, momentos de auge (olas de movilización) y momentos de crisis (fin represivo de un movimiento) forman parte de una misma trayectoria, que debe ser comprendida en su integridad. En particular, esta categoría resulta útil para superar las lecturas deprimentes que surgen cuando un ciclo de movilización culmina en represión y lo que fueron olas de contestación derivan en una desilusión, como diría Aristide Zolberg para graficar esta tendencia, "Post coitum omnia animal triste" (Tarrow, 2009, p. 183).

De esta manera, tanto la década de los sesenta hasta el gobierno de Allende (19701973) como la década de los ochenta, en plena dictadura militar chilena, pueden ser vistas como ciclos de intensa movilización. Los ciclos de movilización son, para Tarrow (2009), calderas de la acción colectiva donde los repertorios -comprendidos como números limitados de desempeños alternativos históricamente establecidos que ligan reivindicaciones a objetos de reivindicación (McAdam, Tarrow \& Tilly, 2009) - se renuevan y se difunden más rápidamente. Ahora bien, los periodos de contracción de la contestación también forman parte del mismo proceso y no necesariamente derivan en el fin de un movimiento social, el cual-por los procesos de reconstrucción que viven los movimientos sociales- bien puede tomar la forma de repliegue a la espera de mejores oportunidades para una nueva acción (Bringel, 2011).

McAdam, Tarrow y Tilly, tratando de hacerse cargo de las críticas por su sesgo estructuralista, así como por su descuido de la dimensión cultural, más recientemente han desarrollado un programa de investigación denominado Contentious Politics, que comprende los movimientos sociales como la forma de coordinación, preparación y mantención de la contestación política como único recurso de las personas comunes contra opositores poderosos. Combinando diversos métodos, teorías de alcance medio, experiencias de movimientos sociales reales, y mediante una perspectiva comparativa, la propuesta de McAdam, Tarrow y Tilly ha enriquecido el campo teórico de los movimientos sociales, así como sus aplicaciones empíricas (Klandermans, Staggenborg \& Tarrow, 2003). Además de su intención de quebrar con la dicotomía entre interés y cultura presente en otras propuestas teóricas, existen conceptos en el programa Contentious Politics que pueden llegar a ser muy útiles para comprender un movimiento como el de los pobladores.

Ahora bien, no se puede dejar de someter esta perspectiva al ejercicio de tematización de sus categorías a la hora de ser aplicadas, más aún si se considera el constante cuestionamiento que hacen los propios autores de esta propuesta conceptual. Además, si esta teoría tiene el mérito de transformar la historia y el tiempo en algo más que meros contextos para los movimientos sociales, a pesar de los esfuerzos de Tilly (2000) 
al final de su carrera, todavía la espacialización de las acciones colectivas y los movimientos sociales continúa siendo un desafío fundamental (Bringel, 2012).

Ahora bien, empleando los conceptos anteriormente expuestos, es posible comprender la población La Victoria como un foco irradiador de repertorios de acción colectiva del movimiento de pobladores. Y, al mismo tiempo, se puede rastrear la trayectoria de este movimiento a partir de la biografía de La Victoria, por su papel protagónico en los diferentes ciclos del movimiento de pobladores, lo que permite superar las lecturas "discontinuistas" de los estudios poblacionales. De manera contraria, La Victoria y el movimiento de pobladores no pueden ser comprendidos sin el espacio como elemento fundante y constituyente del propio movimiento. Y en ese sentido, con mayor o menor mérito, los diferentes estudiosos del movimiento de pobladores poseen una ventaja analítica por sobre la perspectiva de autores como Tarrow o Tilly, que es necesario resaltar.

\section{Consideraciones finales: los pobladores y el derecho a la ciudad}

En el ańo 2004, dos autores (Sabatini \& Wormald, 2004) se preguntaron por lo que quedaba de las movilizaciones por el derecho a la vivienda en Chile y buscaron nuevos elementos de novedad en las luchas populares urbanas de Santiago. Realizando una revisión de la trayectoria del movimiento de pobladores, concluyeron que la integración a la sociedad, y no la transformación social, parece haber sido, y parece ser hoy, el objetivo primordial de los pobladores. En este sentido, para ellos existía una brecha entre el discurso transformador de los dirigentes de los pobladores en las décadas pasadas y la motivación de las bases del movimiento, que no aspiraban a la transformación social, sino que simplemente se adaptaban racionalmente al mecanismo más efectivo para conseguir una casa: la toma de terrenos. La participación popular del movimiento de pobladores aparece aquí como algo meramente instrumental, carente de sentido transformador.

Es por esto que nuevas formas de movilización popular en Santiago, como las luchas en contra de la instalación de vertederos de basuras en la proximidad a una población, son vistas por estos autores como portadoras de semillas de cambio más significativas que las movilizaciones de los sesenta. Las nuevas movilizaciones presen-

tarían formas de ciudadanía con un programa de movilización más autónomo frente al Estado, pues se centrarían en la idea de "calidad de vida" y derecho a la ciudad.

En lo general, su movilización parece encaminada a lograr del Estado acciones y decisiones que respeten sus intereses y que tomen en cuenta sus puntos de vista, los que incluyen destacadamente el "derecho a la ciudad", una demanda que contrasta con la de décadas atrás por el "derecho a la vivienda". Las movilizaciones no se reducen, como en el pasado, a llamados al Estado para que se haga cargo de sus carencias. La lucha actual parece ser, en medida importante, la de quienes buscan ser reconocidos como ciudadanos con plenos derechos, como vecinos de Santiago más que como "pobladores", denominación que en último término los estigmatiza. Es una demanda enarbolada con relativa autonomía frente a los 
partidos políticos y al Estado por distintos grupos y organizaciones populares de Santiago (Sabatini \& Wormald, 2004, p. 74).

La reflexión de estos autores lleva a preguntarse si realmente el movimiento de pobladores adoleció de una noción de derecho a ciudad; al mismo tiempo, cabe cuestionarse si, realmente, la motivación exclusiva del movimiento de pobladores fue la conquista de una casa.

Si bien la vivienda fue la principal reivindicación del movimiento, no fue esta su única motivación. De haberlo sido, los pobladores de La Victoria, una vez que se tomaron sus sitios y obtuvieron sus casas, simplemente se habrían desmovilizado. ¿Por qué los principales focos de resistencia a la dictadura, que determinaron el resurgir del movimiento, como en La Victoria, fueron barrios populares en donde la casa era un conquista de hacía décadas? Tal como señaló el arquitecto Alfredo Rodríguez (1989), "para todos ellos -los pobladores-, resolver su problema de vivienda ha sido mucho más que construir una casa; ha sido construir parte de la ciudad” (p. 9).

Si el derecho a la ciudad puede ser visto solo como derecho a la vida urbana, transformada y renovada, tal como seńaló el propio Lefebvre (2010); y si hoy más que nunca este derecho - como seńala David Harvey (2008) - se encuentra restringido, en la mayoría de los casos, a una reducida elite política y económica que construye y conforma la ciudad de acuerdo con sus propios deseos, entonces ¿no se podría considerar al movimiento de pobladores como una respuesta popular a una forma de construir ciudad de manera excluyente?

Si el espacio, como afirmaba Milton Santos (2008), desde el punto de vista social, tiene rugosidades y no es indiferente a las desigualdades de poder existentes en la sociedad, lo que lo convierte en un campo de fuerzas en permanente disputa, entonces un movimiento social que desde la territorialidad de la población modifica el espacio social, lo puebla, yendo en contra de intereses inmobiliarios o estatales, no puede ser considerado como un movimiento que ignora el derecho a la ciudad.

Si también pensamos el movimiento social como una categoría geográfica, entonces movimiento social y organización del espacio no pueden ser separados. De esta manera:

A expressão movimento social ganha, assim, para a nossa compreensão das identidades coletivas um sentido geográfico muito preciso: é que o vemos como aquele processo através do qual um determinado segmento social recusa o lugar que, numa determinada circunstância espaço-temporal, outros segmentos sociais melhor situados no espaço social pelos capitais (Bourdieu) que já dispõem tentam lhe impor e, rompendo a inércia relativa em que se encontravam, se mobilizam movimentando-se em busca de afirmaçáo das qualidades que acreditam justificarem sua existência (...). Assim o movimento (social) é, rigorosamente, mudança de lugar (social) sempre indicando que aqueles que se movimentam estão recusando o lugar que lhes estava reservado numa determinada ordem de significaçôes (PortoGonçalves, 1999, p. 69). 
Estas consideraciones ayudan a comprender la importancia del movimiento de pobladores para la historia urbana de Santiago de Chile, así como la necesidad de repensar sus categorías, además de permitir valorar la experiencia de la población La Victoria como algo más que un mero ejemplo de conquista de un lugar para vivir. El movimiento de pobladores y La Victoria son testimonio de "una lucha más grande que una casa”, que propuso una forma de territorialidad desde lo popular, indispensable para una ciudad más democrática. oeure

\section{Referencias bibliográficas}

Alonso, A. (2009). As teorias dos movimentos sociais: um balanço do debate. Lua Nova: Revista de Cultura e Politíca, 76, 49-86. doi: 10.1590/S0102-64452009000100003

Amorós, M. (2006). Hasta La Victoria siempre: la lucha por la sobrevivencia durante la dictadura en esta combativa población. Rebelión. Recurso online. Disponible en http:// www.rebelion.org/docs/38023.pdf

Barros, Á. (1978). Juan y La Victoria. Santiago: Ed. Aconcagua.

Bentué, A. (1972). Religión y marginalidad social en la población La Victoria de Santiago (Chile) y reflexión teológica. (Disertación doctoral no publicada). Facultad de Teología Protestante, Universidad de Estrasburgo, Estrasburgo, Francia.

Bringel, B. (2011). A busca de uma nova agenda de pesquisa sobre os movimentos sociais e o confronto político: diálogos com Sidney Tarrow. Política \& Sociedade, 10(18), 51-73. doi: $10.5007 / 2175-7984.2011 \mathrm{v} 10 \mathrm{n} 18 \mathrm{p} 51$

Bringel, B. (2012). Com, contra e para além de Charles Tilly: Mudanças teóricas no estudo das açôes coletivas e dos movimentos sociais. Sociologia \& Antropologia, 02(3), 43-67. Disponible en http://revistappgsa.ifcs.ufrj.br/index.php?area=revista\&revid=13

Cáceres, G. (1993). El movimiento de pobladores de Santiago. 1930-1990. El Mensajero: Boletín del Programa de Educación Popular-CIDE, 55, 1-16.

Cancino, H. (1997). Chile: Iglesia y dictadura 1973-1989. Un estudio sobre el rol político de la Iglesia Católica con el Régimen Militar. Odense, Dinamarca: Odense University Press.

Castells, M. (1972). Chile: Movimiento de pobladores y lucha de clases. Artículo presentado en Primer Encuentro de la Vivienda Social, viexpo, Santiago, Chile.

Centro Interdisciplinario de Desarrollo Urbano (CIDU). (1972). Reivindicación urbana y lucha política: los campamentos de pobladores en Santiago de Chile. EURE, 2(6), 55-82. Disponible en http://www.eure.cl/wp-content/uploads/2010/07/Doc0004 pdf

Comité de Memoria Histórica. (2005). Tortura en poblaciones del Gran Santiago (1973-1990). Santiago: Corporación José Domingo Cañas.

Cortés, A. (2008, mayo). Los comunistas y la toma de terrenos de La Victoria: A 50 años de una de las tomas más grandes de Latinoamérica. Alternativa, 25, 92-101.

Cortés, A. (2009). "Nada por caridad". Toma de terrenos y dictadura: la identidad territorial de la población La Victoria. Tesis para optar al grado de Magíster en Sociología. Instituto Universitário de Pesquisas do Rio de Janeiro (IUPERJ), Río de Janeiro. 
Cortés, A. (2011). Da memória traumática ao relato heróico: O papel da violência na identidade de bairro da población La Victoria em Santiago do Chile. Sociedade e Cultura, 14(2), 357-367. doi: 10.5216/sec.v14i2.17610

De Ramón, A. (2007). Santiago de Chile (1541-1991): Historia de una sociedad urbana. Santiago: Catalonia.

Dubet, F.; Tironi, E.; Espinoza, V. \& Valenzuela, E. (1989). Pobladores: luttes sociales et démocratie au Chile. París: L'Harmattan.

Ducci, M. E. (1997). Chile: el lado oscuro de una política de vivienda exitosa. EURE, 23(69), 99-115. Disponible en http://www.eure.cl/numero/chile-el-lado-obscuro-de-unapolitica-de-vivienda-exitosa/

Espinoza, V. (1982). El movimiento de pobladores: Una evaluación crítica. Proposiciones, 5, 40-52. Disponible en http://bit.ly/18f6voC

Espinoza, V. (1984). Movimiento popular urbano y procesos de institucionalización política. Proposiciones, 11, 57-65. Disponible en http://bit.ly/1acP3BQ

Espinoza, V. (1988). Para una historia de los pobres de la ciudad. Santiago: Ediciones SUR. Disponible en http://www.sitiosur.cl/publicacionescatalogodetalle.php?PID=2649

Espinoza, V. (1994). Tiempos cortos y largos en el movimiento poblacional. Proposiciones, 24, 246-250. Disponible en http://bit.ly/1ckBk8D

Espinoza, V. (1998). Historia social de la acción colectiva urbana: los pobladores de Santiago, 1957-1987. EURE (Santiago), 24(72), 71-84. doi: 10.4067/S025071611998007200004

Fanon, F. (2007). Los condenados de la tierra. México, D.F.: Fondo de Cultura Económica.

Farías, G. (1989). Lucha, vida, muerte y esperanza: historia de la población La Victoria. En A. Rodríguez (Ed.), Constructores de ciudad: nueve historias del primer concurso de "Historia de Poblaciones" (pp. 49-63). Santiago: Ediciones SUR.

Garcés, M. (2002). Tomando su sitio. Santiago: LOM.

Giusti, J. (1973). Organización y participación popular en Chile: El mito del "hombre marginal". Buenos Aires: Facultad Latinoamericana de Ciencias Sociales (Flacso).

Goodwin, J. \& Jasper, J. (1999, marzo). Caught in a winding, snarling vine: the structural bias of political process theory. Sociological Forum, 14(1), 27-54. URL estable: http:// www.jstor.org/stable/685013. Disponible en http://www.jamesmjasper.org/files/Snarling_Vine.pdf

Grupo de Identidad de Memoria Popular (GIMP). (2006). Memorias de La Victoria: relatos de vida en torno a los inicios de la población. Santiago: Quimantú.

Grupo de Trabajo de La Victoria. (2007). La Victoria: Rescatando su historia. Santiago: ARCIS.

Harvey, D. (2008). El derecho a la ciudad. New Left Review, 53, 23-39. Disponible en http:// newleftreview.org/II/53/david-harvey-the-right-to-the-city

Klandermans, B., Staggenborg, S. \& Tarrow, S. (2003). Blending methods and building theories in social movement research. En B. Klandermans \& S. Staggenborg (Eds.), Methods of social movement research (pp. 314-348). Minneapolis, MN: University of Minnesota Press.

La Voz de La Victoria, 1(1), 16 de noviembre de 1958.

La Voz de La Victoria, 1(5), 7 de noviembre de 1959

La Voz de La Victoria, 2(6), 31 de diciembre de 1959.

Lawner, M. (2007). La Victoria: 50 años de victorias. Crítica Cultural, 36, 82-84. 
Lefebvre, H. (2010). O direito à cidade (5ª ed.). São Paulo: Centauro.

Machado da Silva, L. A. (1983). Vida e morte da teoria da marginalidade. En L. Konder, G. Cerqueira Filho \& E. L. Figueiredo (Eds.), Por que Marx? (pp. 217-232). Río de Janeiro: Graal.

McAdam, D., Tarrow, S. \& Tilly, C. (2009). Para mapear o confronto político. Lua Nova, 76, 11-48. Disponible en http://bit.ly/17dXU2t

Millas, O. (1996). Memorias: 1957-1991. Santiago: Ediciones Cesoc (Centro de Estudios Sociales).

Moulian, T. (2002). Chile actual: anatomía de un mito. Santiago: LOM.

Oliven, R. G. (1980). Marginalidad urbana en América Latina. EURE, 19(7), 49-62. Disponible en http://www.eure.cl/numero/marginalidad-urbana-en-america-latina/

Oxhorn, P. (2004). La paradoja del gobierno autoritario: organización de los sectores populares en los ochenta y promesa de inclusión. Politica, 43, 57-83. Disponible en http://www. inap.uchile.cl/images/stories/revistas/politica/43/art-oxhorn.pdf

Paiva, M. (1989). Pasado: Victoria del presente [s.n.]. Santiago.

Pastrana, E. \& Threlfall, M. (1974). Pan, techo y poder: el movimiento de pobladores en Chile (1970-1973). Buenos Aires: Ediciones SIAP.

Pearlman, J. (1977). O mito da marginalidade: favelas e politica no Rio de Janeiro. Río de Janeiro: Paz e Terra.

Pinto, J., Candina, A. \& Lira, R. (1999). Historia contemporánea de Chile, Volumen II: Actores, identidad y movimiento. En G. Salazar \& J. Pinto (Eds.), Historia contemporánea de Chile (Vol. 2). Santiago: LOM.

Porto-Gonçalves, C. W. (1999). A territorialidade seringueira. Geografia e movimento social. GEOgraphia 1(2), 67-88. Disponible en http://bit.ly/19ZFe7C

Quevedo, S. \& Sader, E. (1973). Algunas consideraciones en relación a las nuevas formas de poder popular en poblaciones. EURE, 3(7), 71-81. Disponible en http://bit.ly/14a5CcP

Rodríguez, A. (1989). Presentación. En A. Rodríguez (Ed.), Constructores de ciudad: nueve historias del primer concurso de "Historia de las Poblaciones" (9-11). Santiago: Ediciones SUR.

Sabatini, F. (1981). La dimensión ambiental de la pobreza urbana en las teorías latinoamericanas de la marginalidad. EURE, 8(23), 53-67. Disponible en http://bit.ly/11NJPoc

Sabatini, F. \& Wormald, G. (2004). La guerra de la basura de Santiago: desde el derecho a la vivienda al derecho a la ciudad. EURE, 30(91), 67-86. http://dx.doi.org/10.4067/ S0250-71612004009100005

Salazar, G. (2006). La violencia politica popular en las "Grandes Alamedas": La violencia en Chile 1947-1987 (Una perspectiva histórico popular). Santiago: LOM. [Primera edición: Violencia politica popular en las "grandes alamedas". Santiago de Chile 1947-1987. Vol. I de La violencia en Chile. Santiago: Ediciones SUR, 1990].

Salinas, M. (1996). La recherche d'un lieu sacré: la trajectoire sociale et religieuse des pobladores au Chili (1962-1992). Social Compass, 43(3), 353-366. doi: $10.1177 / 003776896043003005$

Santapau, D. (2005). El evangelio hecho 'acción'. Sacerdotes franceses y pobladores de "La Victoria” (1983-1986). Santiago: Instituto de Historia, Pontificia Universidad Católica de Chile. 
Santa María, I. (1973). El desarrollo urbano mediante los "asentamientos espontáneos": el caso de los "campamentos chilenos". EURE, 3(7), 103-112. Disponible en http://bit. ly/14xyj2a

Santos, M. (2008). Por uma geografia nova (6a ed.). São Paulo: Editora da Universidade de São Paulo (EDUsp).

Schneider, C. (1990). La movilización de las bases, poblaciones marginales y resistencia en Chile autoritario. Proposiciones, 19, 223-243. Disponible en http://bit.ly/11Iy5GN

Tarrow, S. (2009). O poder em movimento: movimentos sociais e confronto político. Petrópolis: Editora Vozes.

Tilly, C. (1995). Los movimientos sociales como agrupaciones históricamente específicas de actuaciones políticas (A. Hynds \& R. May, Trads.). Sociológica, 10(28), 13-36. Disponible en http://www.revistasociologica.com.mx/pdf/2802.pdf

Tilly, C. (2000). Spaces of contention. Mobilization: An International Quarterly, 5(2), 135-159. Disponible en http://bit.ly/16zWwGx

Tironi, E. (1986). La revuelta de los pobladores: integración social y democracia. Nueva Sociedad, 83, 24-32. Disponible en http://nuso.org/upload/articulos/1388_1.pdf

Tironi, E. (Ed.). (1987a). Marginalidad, movimientos sociales y democracia. Proposiciones, 14, 8-20. Disponible en http://www.sitiosur.cl/publicacionescatalogodetalle. php?PID=3399

Tironi, E. (1987b). Pobladores e integración social. Proposiciones, 14, 64-84. Disponible en http://bit.ly/14xIuDU

Touraine, A. (1987). La centralidad de los marginales (conclusión). Proposiciones, 14, 214-224. Disponible en http://www.sitiosur.cl/r.php?id=198\#sthash.V21la3PA.dpuf

Vanderschueren, F. (1971). Pobladores y conciencia social. EURE, 1(3), 95-123. Disponible en http://www.eure.cl/numero/pobladores-y-conciencia-social/

Vekemans, R. \& Silva, I. (1969).El Concepto de marginalidad. En Desal (Centro para el Desarrollo Económico y Social de América Latina), Marginalidad en América Latina: un ensayo de diagnóstico (pp. 15-63). Santiago: Herder.

Vekemans, R. \& Silva, I. (1976). Marginalidad, promoción popular y neo-marxismo. Bogotá: Centro de Estudios para el Desarrollo e Integración de América Latina (CEDiaL).

Verdugo, P. (1985). André de La Victoria. Santiago: Ed. Aconcagua. 